\title{
Choice of Environmental and Economic Path for Building a Supply Chain Financial Cloud Ecosystem under the Background of "Internet +"
}

\author{
Dongchang Zhao \\ School of Economics, Lanzhou University, Lanzhou, 710000 Gansu, China \\ Correspondence should be addressed to Dongchang Zhao; 1169233446@nefu.edu.cn
}

Received 4 February 2021; Revised 18 March 2021; Accepted 20 April 2021; Published 7 May 2021

Academic Editor: Wenqing Wu

Copyright (C) 2021 Dongchang Zhao. This is an open access article distributed under the Creative Commons Attribution License, which permits unrestricted use, distribution, and reproduction in any medium, provided the original work is properly cited.

\begin{abstract}
With the development of the Internet age, the gradual deepening of reforms, and the continuous improvement of marketization, the financial ecological environment is directly related to the risk of financial institutions' loans and the enthusiasm to support local economic development. Capital is accompanied by the physical development and evolution of the production and circulation of goods. After thousands of years of historical development and evolution, it has formulated its own development law and internal logic. In view of the unclear environmental and social evaluation indicators in supply chain research and the model's failure to accurately describe the real supply chain network, an adaptive dynamic relaxation approximation algorithm model is proposed. This model introduces upstream and downstream transit stations as distributors and as a hub between customers, the transportation mode is improved to horizontal and vertical coordinated transportation, and multiple transportation methods are used for transportation. In this paper, the experimental group adopts a new model based on the approximate algorithm of dynamic relaxation factors, and the control group adopts the original old model. The two groups conduct comprehensive data analysis on social indicators, self-indicators, initial indicators, economic indicators, and environmental indicators in each year. Starting from the dimension, comprehensively consider the selection of indicators to measure the level of enterprise performance. The experiment proves that the transportation loss in the green supply chain is between the new model and the old model. When the number of partners is small, the degree of the optimal solution between the new and old models is not obvious, and the degree of optimization of the new model relative to the old model is increasing. It has a significant statistical difference $(P<0.05)$. This shows that efforts to build a good financial ecological environment and an honest and reliable and healthy economic ecological environment are of great importance for promoting the continuous and rapid growth of the social economy.
\end{abstract}

\section{Introduction}

Driven by the new era, the traditional supply chain is slowly transforming into a green supply chain [1]. Traditional enterprise supply chain coordination and management have many shortcomings, such as incomplete communication methods and low utilization efficiency; procurement interaction and tracking methods are performed offline without real-time performance; enterprises and suppliers are not connected. While understanding the development of our country's software and information technology service industry, there is a research on the impact of supply chain integration on the performance of enterprises in the industry. According to the concept and characteristics of supply chain integration, the relevant factors that influence the methods of evaluation and evaluation of business performance determine the supply chain integration and the indicators of measuring the performance of companies and define relevant models. This document uses existing data from listed companies in the software services and information technology industry to separate supply chain integration into customer integration and supplier integration, exploring the relationship between customer integration, vendor integration, and corporate performance.

There have been research results in many aspects of green supply chains abroad. In terms of green supply chain model 
collaboration models, although the design models are very different, the overall direction remains the same. The supply chain collaboration model of green supply chain is an efficient collaboration. The mode is to meet customer order needs through good materials and commodities. Azzi et al. proposed a new type of supply chain network collaboration planning model and found that the total profit level of supply chain nodes is positively correlated, and the two-way collaborative green supply chain is optional [2]. Lin CS and Lin CY use a multistage Stackelberg game method to study the green procurement relationship between manufacturers and suppliers in the supply chain and effectively reduce suppliers' carbon emissions [3]. Lin CS and Lin CY studied the asymmetric duopoly model of two competing supply chains under different carbon emission technologies and encouraged the use of clean energy technologies to reduce carbon emissions $[3,4]$. Papert and Pflaum study the production and emission reduction decision-making problems of an order-based supply chain composed of manufacturers and retailers under the constraints of the capital market and trade market [5].

In recent years, there have been more and more researches on the green supply chain in China. The logistics technology in the green supply chain is also the focus of the supply chain research. The ultimate goal of the green logistics technology is the sustainable development of the ecological environment [6]. Development is not only for economic benefits, but also environmental benefits and social benefits, and it is the unity of these benefits. Papert and Pflaum's research focuses on carbon dioxide emissions in the supply chain, from freight energy use to inventory storage, and establishes a supply chain model based on discrete event simulation [5]. Stolze et al. studied the dual-objective, multistage, multicommodity mixed integer nonlinear programming model considering the impact of different vehicle and raw material quality on the total cost and carbon dioxide emissions of the green supply chain and finally explained and solved the method through constraint numerical examples [7]. Christensen et al. use a hybrid life cycle assessment method to generate the carbon emissions of a monocrystalline silicon photovoltaic system and use a multiregion inputoutput model to ensure the integrity of the system boundary [8]. Souza et al. studied the decision-making of low-carbon supply chain and coordinated low-carbon manufacturers to produce a product and approved investment in green technology to reduce carbon emissions in production applications $[9,10]$.

This article mainly focuses on the optimization design of the multilayer structure of the green supply chain of small and medium manufacturing enterprises at the economic, social, and environmental levels. When studying the impact of supply chain integration on corporate performance, we comprehensively consider the selection of indicators to measure corporate performance from multiple dimensions, breaking through the limitation that most scholars in the past only use financial indicators to represent the impact of supply chain integration on corporate performance. Explore the impact of supply chain integration on corporate performance and drawing conclusions, judge the relationship between supply chain management and corporate perfor- mance in our country's software and information technology services sector, and strengthen corporate governance and improve corporate performance [11].

\section{Choice of Environmental Economic Path for Constructing a Supply Chain Financial Cloud Ecosystem}

2.1. Financial Ecological Environment Index and Factor Analysis Method. Since each principal factor rotates the original variables by the data transformation matrix to make them independent of each other, then the comprehensive evaluation value of the principal factors is calculated, thereby eliminating the correlation images between the indicators $[12,13]$. In factor analysis, the weight of each major factor is not determined and is determined by the percentage of the major factor that determines uterine change. This overcomes the shortcomings of certain evaluation methods that define the entire book and makes the evaluation results more objective and logical $[14,15]$. Steps of factor analysis evaluation method are as follows:

(1) Build the original data matrix

Set $n$ samples, and each sample has $p$ indicators, so the original data matrix is obtained:

$$
X=\left[\begin{array}{cccc}
X_{11} & X_{12} & \cdots & X_{1 p} \\
X_{21} & X_{22} & \cdots & X_{2 p} \\
\cdots & \cdots & \cdots & \cdots \\
X_{n 1} & X_{n 2} & \cdots & X_{n p}
\end{array}\right]
$$

(2) Standardize the transformation of the original data

In order to avoid the influence of differences and the order of the measured values and variables, it is necessary to standardize the data first to make the indicators comparable [16]. The specific standardized formula is as follows:

$$
Y_{i j}=\frac{\left(X_{i j}-E X_{j}\right)}{\sqrt{D X_{j}}} .
$$

Among them,

$$
\begin{aligned}
& E X_{j}=\frac{1}{n} \sum_{i=1}^{n} X_{i j}, \\
& D X_{j}=\frac{1}{n-1} \sum_{i=1}^{n}\left(X_{i j}-E X_{j}\right)^{2} .
\end{aligned}
$$

In the above formula, $i=1,2,3, \cdots, n, j=1,2,3, \cdots, 18$, and $X_{i j}$ are the index values before standardization, and $Y_{i j}$ is the index value after standardization. With certain performance improvements, there are new requirements for general adaptability and specific adaptability. The main problem with basketball gymnastics in Japan is that there are too many regular gymnastics, special gymnastics are not covered, and the obtained physical condition cannot be used for special gymnastics. 
(3) Calculate the correlation coefficient matrix $R$

Let $r_{i j}$ be the correlation coefficient between index $i$ and index $j$ after standardization; then,

$$
\begin{gathered}
r_{i j}=\frac{\operatorname{COV}\left(Y_{i}, Y_{j}\right)}{\sqrt{D Y_{i} D Y_{j}}}=E Y_{i} Y_{j}, \\
R=\left[\begin{array}{cccc}
r_{11} & r_{12} & \cdots & r_{1 p} \\
r_{21} & r_{22} & \cdots & r_{2 p} \\
\cdots & \cdots & \cdots & \cdots \\
r_{n 1} & r_{n 2} & \cdots & r_{n p}
\end{array}\right] .
\end{gathered}
$$

(4) Find the characteristic root, characteristic vector, and contribution rate of the correlation matrix $R$

Human motion tracking based on template matching currently primarily uses error metrics between two matching pixel blocks. There are three main error metrics based on block matching, an error metric based on a crosscorrelation function and a normalized mean square. According to characteristic equation $|R-\lambda I|=0,|R-\lambda I|=$ 0 characteristic roots $\lambda_{j}(j=1,2, \cdots, p)$ are obtained, and Formula (7) can be obtained.

$$
\left(R-\lambda_{j} I\right) \mu_{j}=0 .
$$

Find the feature vector $\mu_{j}(j=1,2, \cdots, p)$ corresponding to the feature root $\lambda_{j}$.

(5) Determine the cumulative variance contribution rate of the main factor

The contribution rate of the $i$ factor is $d_{i}=\lambda_{i} / \sum_{i=1} \lambda_{i}$, that is, the variation degree of each factor accounts for the percentage of the overall matrix variation degree.

(6) Calculate factor score and total score

$$
F(a)=\lambda_{1} f_{a 1}+\lambda_{2} f_{a 2}+\cdots+\lambda_{m} f_{a m} .
$$

Among them,

$$
f_{i}=A^{T} R^{-1} X_{a}
$$

\subsection{Dynamic Relaxation Approximation Algorithm}

2.2.1. Traditional Particle Swarm Algorithm. The maximum communication delay that the user can tolerate under the cloud and fog architecture is guaranteed, and the user's request is processed within the acceptable communication delay [17]. The initial settings of the particle swarm algorithm will affect the search performance of the particle swarm algorithm. The more individual particles, the larger the search range of the particle swarm, the easier it is to get the best approximate solution close to the optimal solution, and the search speed of the particles is too fast. It is easy to fall into the local optimal solution $[18,19]$. Therefore, it is of theoretical and practical importance for the application of the particle swarm algorithm to generate the initial particle swarm in an appropriate manner. The single-particle tree structure coding design emphasizes the connection between upstream and downstream in the supply chain network. The initial position encoding method of individual particles is as Formula (10), and the initial velocity encoding method is as Formula (11).

$$
\begin{aligned}
& X_{\text {in }}^{t}=\left(X_{i 1}^{t}, X_{i 2}^{t}, \cdots, X_{i D}^{t}\right), i \in I, n \in D, t \in T, \\
& V_{\text {in }}^{t}=\left(V_{i 1}^{t}, V_{i 2}^{t}, \cdots, V_{i D}^{t}\right), i \in I, n \in D, t \in T .
\end{aligned}
$$

In constructing the initial particle swarm, let $D$ be the spatial dimension of individual particles in the particle swarm, the value of $D$ will affect the search ability of the particle swarm, let $T$ be the maximum number of iterations of the algorithm, and $T$ is the number of search particles in each individual particle $[20,21]$.

For all search speeds that exceed the speed range area, change all cross-border speeds to the maximum or minimum search speeds. The particle value range and speed range are shown in Formulas (12) and (13).

$$
\begin{array}{r}
\left\{\begin{array}{l}
X_{\text {in }}^{t}\left(X_{\text {in }}^{t} \geq X_{\text {max } \_n}\right)=X_{\text {max } \_n}, n \in D, \\
X_{\text {in }}^{t}\left(X_{\text {in }}^{t} \leq X_{\text {min } \_n}\right)=X_{\text {min } \_n}, n \in D,
\end{array}\right. \\
\left\{\begin{array}{l}
V_{\text {in }}^{t}\left(V_{\text {in }}^{t} \geq V_{\text {max } \_n}\right)=V_{\text {max } \_n}, n \in D, \\
V_{\text {in }}^{t}\left(V_{\text {in }}^{t} \leq V_{\text {min } \_n}\right)=V_{\text {min } \_n}, n \in D .
\end{array}\right.
\end{array}
$$

The initial speed of the particle swarm has a great influence on the convergence speed of the algorithm. If the speed is set too fast, the optimal solution of the algorithm will easily exceed the optimal solution, and if the initial speed is set too slow, it will easily fall into the local optimum.

2.2.2. Lagrangian Relaxation Algorithm. The complex constraints that make the final problem difficult are added to the objective function as penalty conditions, so that the objective function is easy to solve, and the constrained optimization problem can be transformed into an unconstrained optimization problem [22]. In the process of solving the throat relaxation of the objective function, the objective function can always remain linear. The model description of the original problem is shown in Formula (14):

$$
Z_{I P}=\min C^{T} X, \text { s.t. } A X \geq b, C X \geq d, X \in Z_{n}^{+} .
$$

Lagrangian relaxation transformation is performed on the original problem to obtain a Lagrangian linear model, as shown in Formula (15):

$$
Z_{L P}=\min C^{T} X+\mu^{T} \times(b-A X) \text {, s.t. } C X \geq d, X \in Z_{n}^{+} .
$$

In the above formula, $\mu$ is the Lagrangian relaxation factor, and the initial value cannot be defaulted to 0 . The calculation is shown in Formula (16):

$$
\mu^{i+1}=\max \left\{\mu^{i+1}+\operatorname{step}^{i} \times \operatorname{sub}^{i}, 0\right\} .
$$


The solution of the subgradient of Lagrangian relaxation is shown in Formula (17):

$$
\operatorname{sub}^{i}=\partial Z_{U P}\left(X^{i}\right)
$$

In the formula, $Z_{U P}$ is the boundary value of $Z_{L P}$, which is generally the maximum or minimum value of the current iteration. Select the step size. The step size affects the convergence of the relaxation algorithm iteration. If the step size is too short, the algorithm will not be able to get the optimal solution. If the step size is too long, the calculation will exceed the extreme value and the settlement result will diverge. Therefore, the relaxation algorithm of the step length needs to take a gradually reduced value. The calculation of the step length step is shown in Formula (18):

$$
\operatorname{step}^{i}=\frac{Z_{U P}\left(X^{i}\right)-Z_{L P}\left(X^{i}\right)}{\left\|\operatorname{sub}^{i}\right\|_{2}^{2}} .
$$

2.2.3. Dynamic Relaxation Approximation Algorithm. According to the initial distribution of individual particles $X$, it is necessary to determine whether each supplier and manufacturer has joined the production operation in the current distribution plan [23]. The constraints of suppliers and manufacturers determine the corresponding supply of individual particles whether the company and the manufacturer are operating $[24,25]$, and the corresponding location decision formula for the company is shown in Formulas (19) and (20):

$$
\begin{gathered}
O S_{i}=\left\{\begin{array}{l}
0, \text { if } \cdot \sum_{j=1}^{J} \sum_{m=1}^{M} A d c p_{i j m}=0, \\
1, \text { if } \cdot \sum_{j=1}^{J} \sum_{m=1}^{M} A d c p_{i j m}>0,
\end{array}\right. \\
O P_{i}=\left\{\begin{array}{l}
0, \text { if } \cdot \sum_{k=1}^{K} \sum_{n=1}^{N} A d c p_{j k n}=0, \\
1, \text { if } \cdot \sum_{k=1}^{K} \sum_{n=1}^{N} A d c p_{j k n}>0 .
\end{array}\right.
\end{gathered}
$$

Every time the particle swarm is updated, the location decisions of suppliers and manufacturers need to be updated as the particle swarm changes.

\subsection{Financial Ecology and Financial Ecological Environment}

2.3.1. Ecological and Ecological Characteristics. The economic environment is the most basic environment. It is the basis for the survival of the financial system and determines the degree of development of the financial system. The financial system was created after economic growth reached a certain level and developed simultaneously with economic growth [26]. Traditional financial theory understands financing as a tool and understands financing as the external and dependent elements of the economy and essentially separates the inevitable internal links between the financial system and the economic

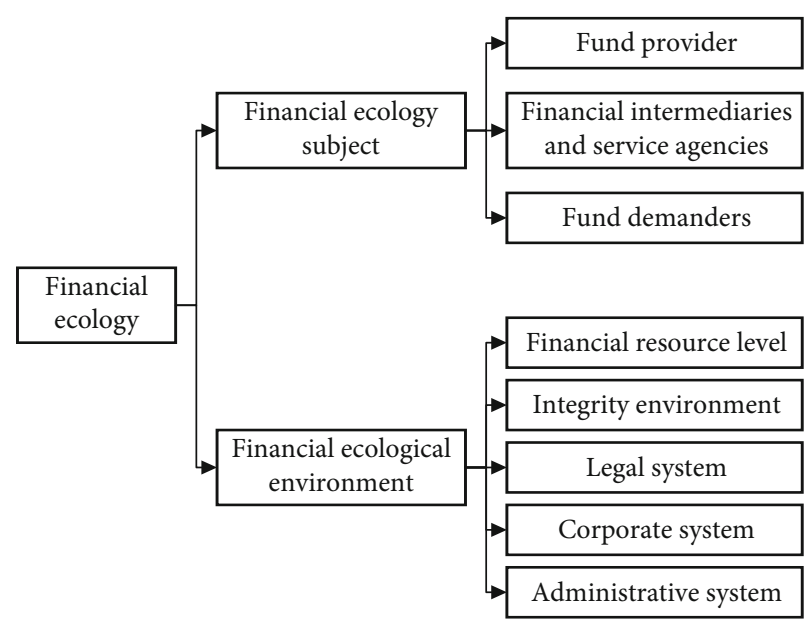

FIgURE 1: Relationship between financial ecology and financial ecology environment.

environment $[27,28]$. Modern financial theory believes that finance is inherent in the economy, and the economy is increasingly globalized and spread to the overall economy. Finance has become the economy itself and the core of the modern economy. The relationship between the financial system and the economic environment is that the economic environment determines the financial growth and the limits of financial growth or promotes economic growth. Laws and regulations are relatively stable, authoritative, and coercive. At this point, policies cannot be compared with them. However, the flexibility and timeliness of policies are stronger than those of laws and regulations. The characteristics of the policy determine that when judging the policy environment, it is necessary to adopt different standards from judging the legal environment $[23,29,30]$.

2.3.2. The Connotation of Financial Ecological Environment. The law must be able to effectively protect the interests of investors and creditors, to contribute to the establishment and maintenance of good economic order, and thus to ensure the smooth and efficient operation of the financial system. A well-developed credit system can not only effectively reduce the cost of information collection, but also reduce the negative choice and moral hazard caused by information asymmetry and reduce the occurrence of financial gaps and financial crises. At present, in order to improve the regional financial ecological environment, many regions are actively preparing to build financial security zones [31, 32]. However, improving the financial ecological environment cannot simply be equated with building a financial safety zone, and these two concepts cannot be confused with each other. Therefore, the construction of a financial security zone cannot be equated with improving the financial ecological environment.

\subsubsection{Financial Ecology and Financial Ecological Environment.}

The financial ecosystem includes financial institutions and financial markets that provide direct financial products and financial services, as well as individuals, enterprises, and government agencies that consume various financial products and financial services, as well as those who formulate policies 
TABLE 1: Data sheet of evaluation index system for index reliability testing.

\begin{tabular}{|c|c|c|c|c|c|c|}
\hline & Very clear & Clear & General & Not clear & Chaotic & Alpha \\
\hline Social indicators & 0.307 & 0.388 & 0.174 & 0.083 & 0.048 & 0.9064 \\
\hline Self index & 0.191 & 0.241 & 0.253 & 0.189 & 0.146 & 0.8433 \\
\hline Initial index & 0.224 & 0.284 & 0.229 & 0.177 & 0.086 & 0.8169 \\
\hline Economic indicators & 0.192 & 0.176 & 0.312 & 0.184 & 0.136 & 0.7672 \\
\hline Environmental indicators & 0.217 & 0.208 & 0.244 & 0.194 & 0.137 & 0.7394 \\
\hline
\end{tabular}

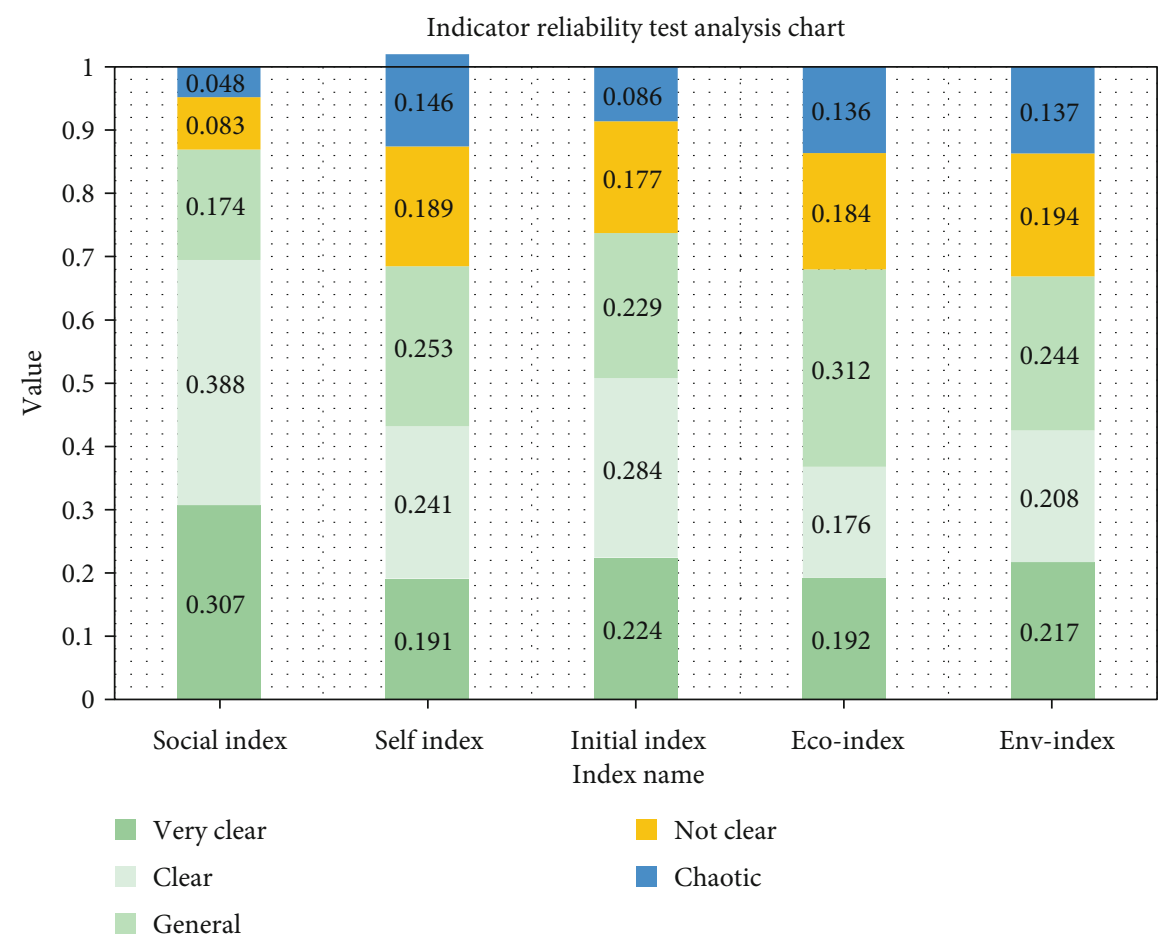

FIgURE 2: Indicator reliability test analysis chart.

and regulate financial services. The implementation of supervision is a function that directly affects the operation of financial institutions and financial markets. It is also a financial decision-making agency and financial regulatory agency that directly affect the type, price, and quality of financial products and services [33]. Figure 1 can clearly show the relationship between the financial ecology and the financial ecological environment.

A comprehensive survey of the survival and development status of the elements of financial entities is important, and without any of these elements, it is difficult to achieve realistic financial transactions. Therefore, it is difficult to fully understand the connotation of financial ecology only from the perspective of financial organization, ignoring the living conditions of other financial entities. Like the natural ecosystem, financial ecology is deeply influenced by people. However, the economic transactions and social interactions brought about by people, as well as the spillover effects produced in this process, make the financial ecological environment more complicated than the natural ecological environment $[34,35]$. Human activities not only involve producers, consumers, and decomposers, but also strongly affect economic systems, social systems, laws and regulations, and culture. The relationship
TABLE 2: Social indicator preprocessing data table.

\begin{tabular}{ccccc}
\hline Year & Old model & New model & Optimal solution & $P$ \\
\hline 2016 & 1.92 & 1.28 & 1.14 & 0.032 \\
2017 & 2.57 & 2.23 & 2.03 & 0.027 \\
2018 & 4.72 & 4.26 & 3.97 & 0.019 \\
2019 & 6.33 & 5.86 & 5.59 & 0.010 \\
2020 & 14.95 & 13.18 & 12.76 & 0.012 \\
\hline
\end{tabular}

between them is frequent, diverse, and complex. Therefore, it is very difficult and unnecessary to absolutely distinguish between financial ecology and financial ecology.

\section{Experimental Design of Environmental Economic Path Selection for Constructing Supply Chain Financial Cloud Ecosystem}

3.1. Data Sources. In terms of data selection, to ensure the authenticity and authority of the data, this article mostly uses official data or authoritative research results. The data mainly come from 2016-2020 China Statistical Yearbook, China 


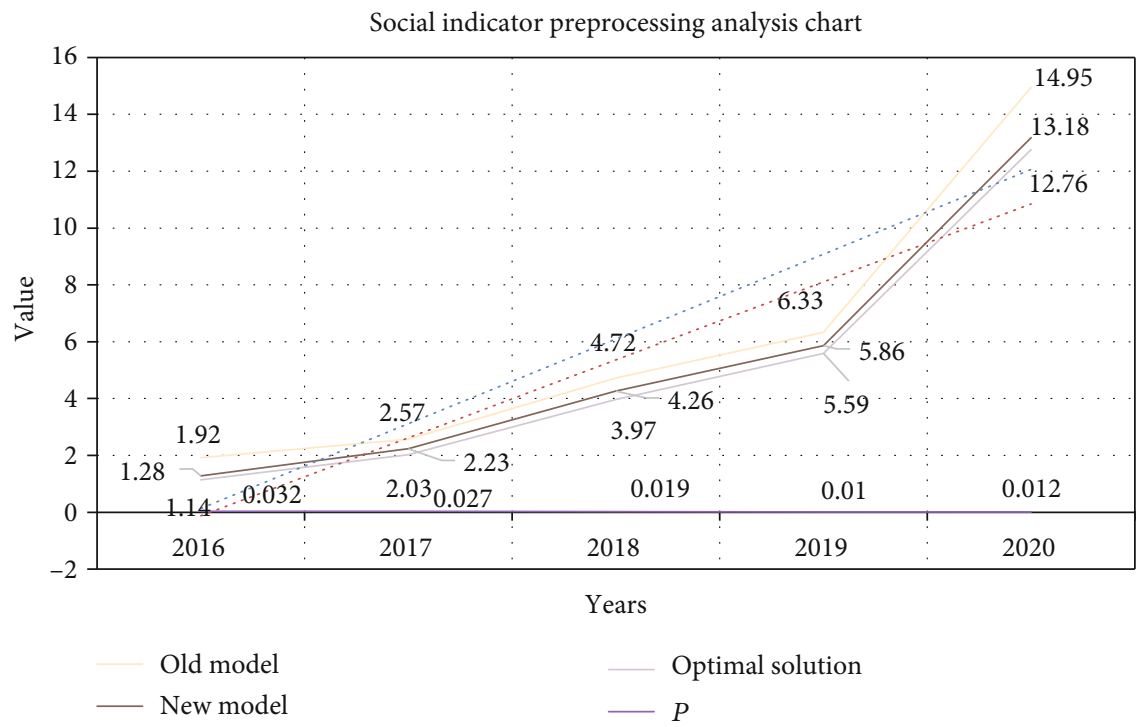

FIGURE 3: Social indicator preprocessing analysis chart.

Regional Economic Statistical Yearbook, financial operation report, and other relevant statistical data. Combining multiparty statistical data from financial institutions and their regulatory agencies, the evaluation index data is collected through multiple channels, and considering that price factors have a greater impact on the fitting results, all selected indicators are processed at constant prices. The experimental group adopts a new model based on the approximate algorithm of dynamic relaxation factors, and the control group adopts the original old model. The two groups conduct comprehensive data analysis on social indicators, self indicators, initial indicators, economic indicators, and environmental indicators for each year. Set out to comprehensively consider the selection of indicators to measure the level of corporate performance, explore the impact of supply chain integration on corporate performance, and summarize conclusions.

3.2. Experimental Method. The green supply chain model of manufacturing companies is more complex in terms of the spatial complexity of the model. The cooperation between the partners is not only the initial horizontal cooperation, but also the vertical cooperation relationship. For different numbers of partners, transit stations, customers, and different customer order numbers, the approximate dynamic relaxation factor algorithm is used to calculate the weight values of various indicators in the construction company's supply chain, and then, cplex is used to solve the problem.

3.3. Data Preprocessing. A benchmark value is anchored for each index collected, and the standardized score obtained by each year's index on this index depends on the proportional relationship between the original value of the city index and the anchored benchmark value.

Regarding positive indicators (the higher the value, the better the indicator), the standardized score $=$ the original value of the indicator/anchored reference value.
TABle 3: Environmental index preprocessing data sheet.

\begin{tabular}{lccccc}
\hline Year & $\begin{array}{c}\text { Water } \\
\text { pollution }\end{array}$ & $\begin{array}{c}\text { Air } \\
\text { pollution }\end{array}$ & $\begin{array}{c}\text { Noise } \\
\text { pollution }\end{array}$ & $\begin{array}{c}\text { Total } \\
\text { pollution }\end{array}$ & $P$ \\
\hline 2016 & 2.56 & 3.08 & 3.66 & 3.41 & 0.017 \\
2017 & 2.72 & 2.90 & 3.69 & 3.37 & 0.014 \\
2018 & 2.69 & 2.92 & 3.35 & 3.48 & 0.011 \\
2019 & 2.42 & 3.25 & 3.15 & 3.50 & 0.008 \\
2020 & 2.43 & 2.92 & 3.16 & 3.55 & 0.005 \\
\hline
\end{tabular}

Regarding the inverse index (the smaller the value, the better the index), the standardized score $=$ anchored benchmark value/the original value of the index.

3.4. Statistical Data Processing Method. The SPSS23.0 software was used for data processing, and the count data was expressed in percentage (\%), $k$ is the number of data in this experiment, $\sigma^{2}$ is the variance of all survey results, and $P<0.05$ indicates that the difference is statistically significant. The formula for calculating reliability is shown in Formula (21).

$$
a=\frac{k}{k-1}\left(1-\frac{\sum \sigma_{i}^{2}}{\sigma^{2}}\right) .
$$

\section{Experimental Choice of Environmental Economic Paths for Constructing Supply Chain Financial Cloud Ecosystem}

4.1. Evaluation Index System Based on Index Reliability Testing. Reliability refers to the stability and reliability of the questionnaire. This article adopts the $\alpha$ coefficient method created by L.J. Cronbach. The $\alpha$ coefficient can be obtained by Reliability Analysis in SPSS software. It is generally believed that the $\alpha$ coefficient above 0.8 indicates that the effect of the index setting is very good, and above 0.7 is also 


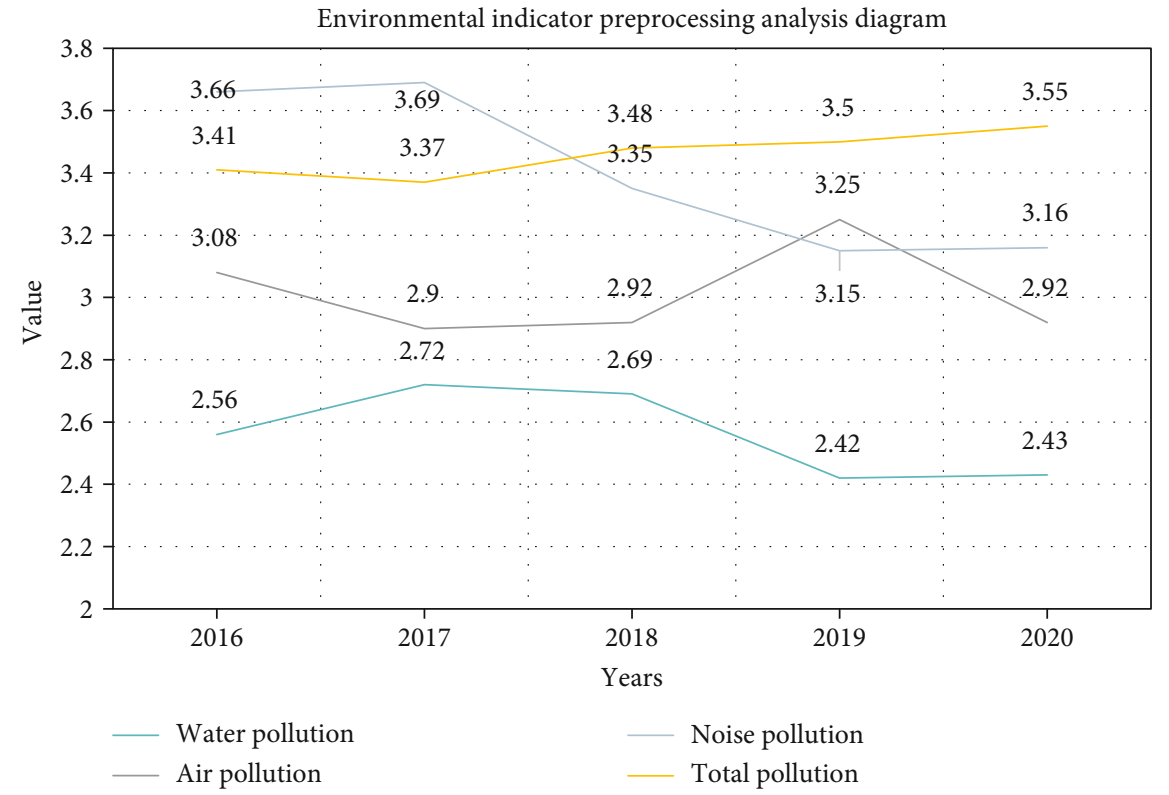

FIGURE 4: Environmental indicator preprocessing analysis diagram.

acceptable. Here, we analyze the reliability of each type of object, and the reliability index we choose for each type of object is slightly different. The results are shown in Table 1 .

It can be seen from Figure 2 that the data obtained from social indicators, own indicators, initial indicators, economic indicators, and environmental indicators have an acceptable impact on this experiment $(\alpha>0.7)$, which can be analyzed from the side in the comparative analysis of the subitem evaluation indicators of the financial ecological environment of the supply chain of various cities in our country, and the regional economic development and financial foundation are not balanced.

\subsection{Based on a Single Indicator}

4.2.1. Analysis Based on Social Indicators. Starting from multiple dimensions, comprehensively consider the selection of indicators to measure the level of corporate performance, explore the impact of supply chain integration on corporate performance, and summarize conclusions. Here, we have collected social indicator data through various channels from 2016 to 2020. After preprocessing these data, we analyze the preprocessed data. The results are shown in Table 2.

Figure 3 uses a line chart to describe the social indicators in the economic aspects of the green supply chain of manufacturing companies and compares the social indicators between the new model and the old model with the optimal solution. At first, the optimization effect of the new model is not obvious. As the degree of marketization continues to increase, the approximate algorithm of dynamic relaxation factors is used to deal with partners, and the model optimization effect with transit stations becomes more and more obvious, and it is close to the value obtained from the optimal solution $(P<0.05)$.

4.2.2. Analysis Based on Environmental Indicators. For the environmental aspects of the green supply chain, including
TABLE 4: Initial index preprocessing data table.

\begin{tabular}{cccccc}
\hline Year & $\begin{array}{c}\text { Rail } \\
\text { transport }\end{array}$ & $\begin{array}{c}\text { Road } \\
\text { transport }\end{array}$ & $\begin{array}{c}\text { Number of } \\
\text { partners }\end{array}$ & $\begin{array}{c}\text { Customer } \\
\text { order }\end{array}$ & $P$ \\
\hline 2016 & 2.65 & 3.05 & 3.27 & 3.41 & 0.005 \\
2017 & 2.56 & 3.30 & 3.17 & 3.76 & 0.005 \\
2018 & 2.91 & 3.21 & 3.64 & 3.64 & 0.005 \\
2019 & 2.92 & 3.01 & 3.51 & 3.35 & 0.005 \\
2020 & 2.97 & 3.03 & 3.05 & 3.56 & 0.005 \\
\hline
\end{tabular}

water pollution, carbon emissions, and noise pollution, multiply the corresponding weight values to calculate the governance cost indicators of the old model and the new model. Here, we have collected environmental indicator data through multiple channels from 2016 to 2020 . After preprocessing these data, we analyze the preprocessed data. The results are shown in Table 3.

Figure 4 linearly describes the data of transportation losses in the green supply chain between the new model and the old model. When the number of partners is small, the degree of new and old model in relation to the optimal solution is not obvious. When the number of partners is large, the new model's model optimization effect is slightly reduced. As the number of partners gradually increased, the degree of optimization of the new model compared to the old model became more and more evident $(P<0.05)$.

4.2.3. Analysis Based on Initial Indicators. Starting from multiple dimensions, comprehensively consider the selection of indicators to measure the level of corporate performance, explore the impact of supply chain integration on corporate performance, and summarize conclusions. Here, we have collected the initial indicator data through various channels from 2016 to 2020. After preprocessing these data, we analyze the preprocessed data. The results are shown in Table 4. 


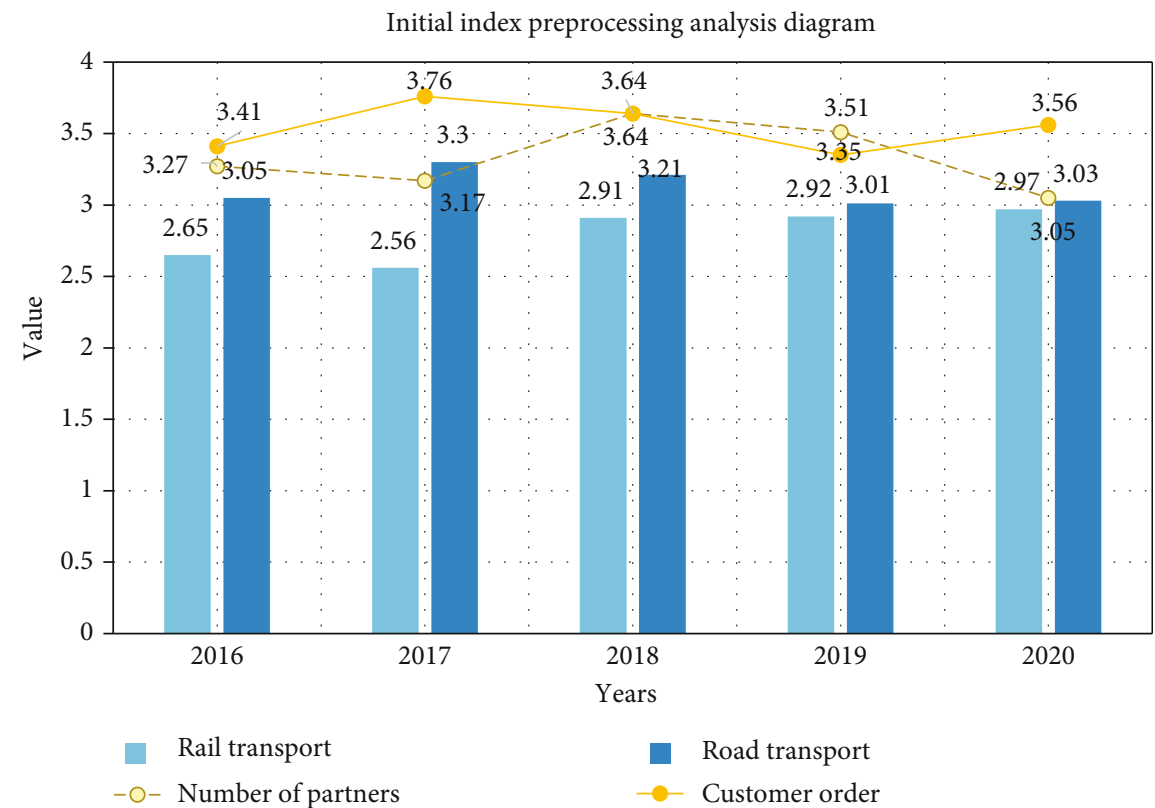

Figure 5: Initial index preprocessing analysis diagram.

TABLE 5: Economic index preprocessing data table.

\begin{tabular}{|c|c|c|c|c|c|c|}
\hline Year & Transportation cost & Other expenses & Transportation time & Transport distance & Breakdown cost & $P$ \\
\hline 2016 & 2.01 & 2.88 & 3.29 & 3.57 & 3.31 & 0.024 \\
\hline 2017 & 2.46 & 2.69 & 2.99 & 3.16 & 3.56 & 0.029 \\
\hline 2018 & 2.11 & 2.64 & 2.95 & 3.50 & 3.67 & 0.032 \\
\hline 2019 & 2.26 & 2.65 & 2.94 & 3.52 & 3.34 & 0.017 \\
\hline 2020 & 2.33 & 2.38 & 3.19 & 3.51 & 3.31 & 0.021 \\
\hline
\end{tabular}

Figure 5 depicts the comparison of the accident rate in the green supply chain between the new model and the old model, because the introduction of rail transport in the new model has generally reduced the accident rate of road transport. With the gradual increase in the number of partners and customer orders, the optimization degree of the new model relative to the old model has become more and more obvious $(P<0.05)$.

4.2.4. Analysis Based on Economic Indicators. Starting from multiple dimensions, comprehensively consider the selection of indicators to measure the level of corporate performance, explore the impact of supply chain integration on corporate performance, and summarize conclusions. Here, we have collected economic indicator data through various channels from 2016 to 2020. After preprocessing these data, we analyze the preprocessed data. The results are shown in Table 5.

It can be seen from Figure 6 that in terms of economic indicators, the comparison between the new model using the approximate algorithm of the dynamic relaxation factor and the old model shows that the processing effect of the new model is better, even better than the optimal solution $(P<0.05)$, which shows that the integration of suppliers as a whole has a significant moderating effect on improving the performance of enterprises. The obvious reasons for the results may be caused by the characteristics of the industry and the high degree of dependence on suppliers by enterprises in the industry.

4.2.5. Analysis Based on Own Indicators. Starting from multiple dimensions, comprehensively consider the selection of indicators to measure the level of corporate performance, explore the impact of supply chain integration on corporate performance, and summarize conclusions. Here, we have collected our own indicator data through multiple channels from 2016 to 2020. After preprocessing these data, we analyze the preprocessed data. The results are shown in Table 6.

It can be seen from Figure 7 that for its own indicators, the comparison between the new model using the dynamic relaxation factor approximation algorithm and the old model shows that the processing effect of the new model is better $(P<0.05)$, which shows that the method is not only useful, and the next algorithm reduces the complexity of the space, reduces the operating time, and improves the efficiency of the supply chain to solve the partners. And as the number of partners increases, the faster the improvement algorithm runs, the more obvious the benefits. 


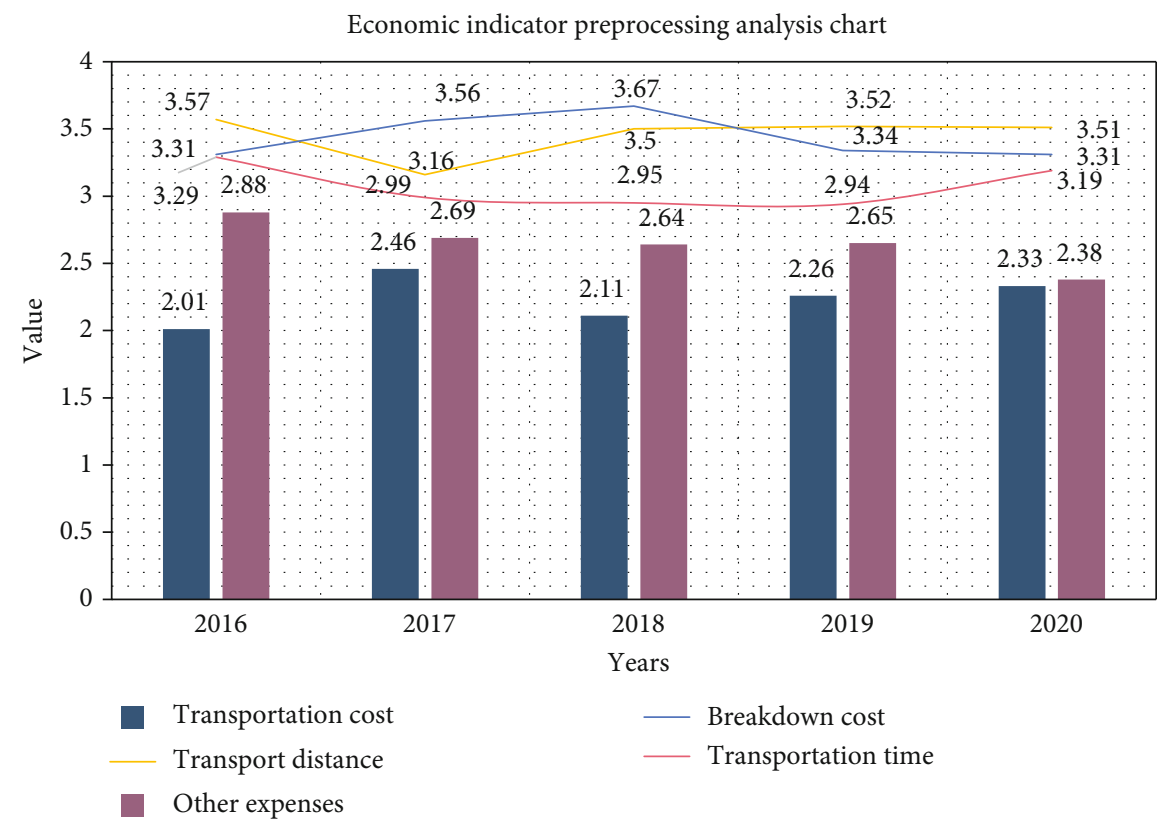

FIGURE 6: Economic indicator preprocessing analysis chart.

TABLE 6: Self index preprocessing data table.

\begin{tabular}{|c|c|c|c|c|c|c|}
\hline Year & Operating cost & Operating loss & Raw material cost & Operational pollution cost & Carbon allowance & $P$ \\
\hline 2016 & 2.12 & 2.31 & 3.15 & 3.17 & 3.49 & 0.001 \\
\hline 2017 & 2.54 & 2.55 & 3.10 & 3.20 & 3.78 & 0.002 \\
\hline 2018 & 2.29 & 2.31 & 3.01 & 3.22 & 3.76 & 0.002 \\
\hline 2019 & 2.43 & 2.59 & 3.20 & 3.58 & 3.77 & 0.001 \\
\hline 2020 & 2.06 & 2.73 & 3.20 & 3.65 & 3.94 & 0.001 \\
\hline
\end{tabular}

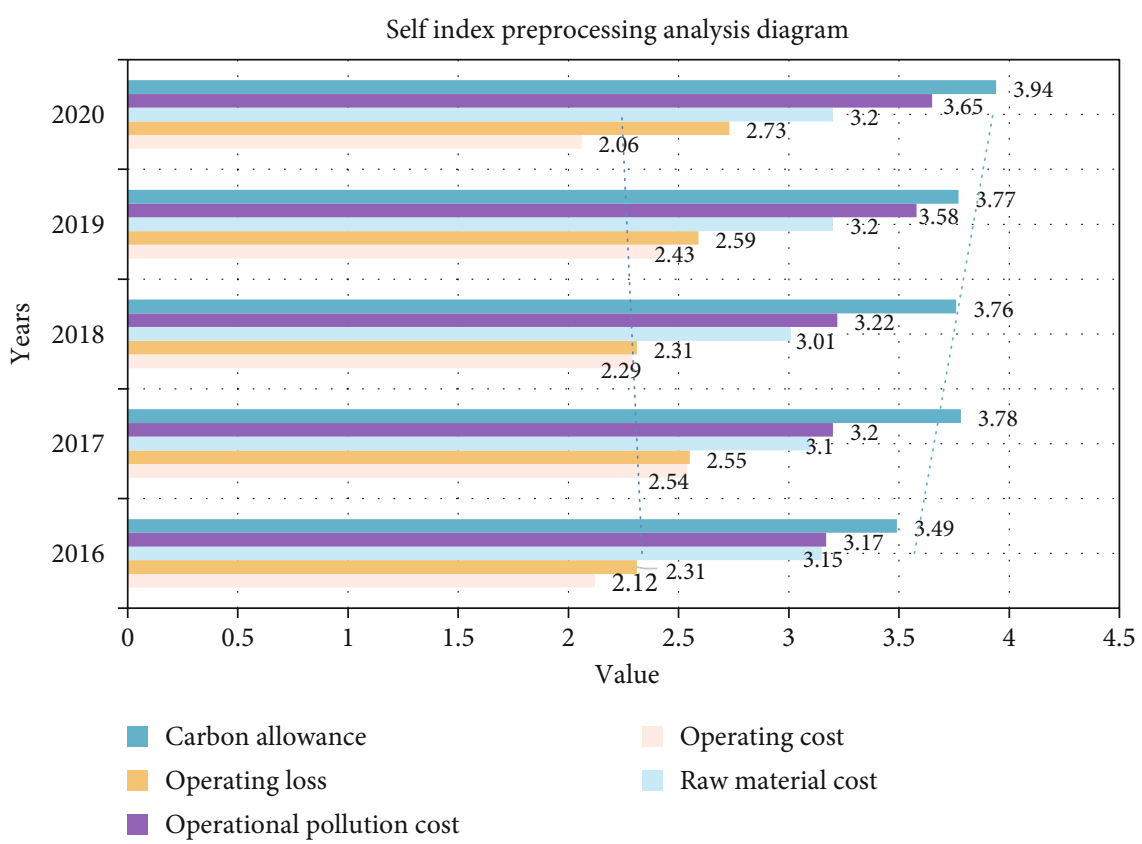

FIGURE 7: Self index preprocessing analysis diagram. 


\section{Conclusions}

On the basis of studying the optimization methods of various green supply chain mathematical models at home and abroad, this paper designs a new green supply chain model for small and medium manufacturing enterprises based on the compound weight method. The newly introduced coordinated transportation method improves transportation efficiency, reduces transportation time, and reduces transportation accident rate. In the four-level network architecture of the traditional supply chain, upstream and downstream transfer stations are newly introduced to integrate the volume of vehicles that are not fully loaded. The upstream transfer station to the downstream transfer station is railway transportation, which reduces transportation costs and increases the supply chain. The complexity of the model effectively innovates the structure of the model. The improved supply chain model with transit can effectively deal with the problem of green supply chain network optimization. In the study of small and medium-sized green supply chain examples, the improved index solution quality of the transit supply chain model is significantly better than that of the nontransit supply chain model. And as the scale of the calculation example increases, the optimization effect of each index becomes more obvious.

The government should be the leader in the construction of financial ecological environment. When formulating economic plans, it should start with improving the regional economic environment, based on resource advantages, adapt measures to local conditions, and formulate economic development plans around market reforms, so as to create a harmonious economic environment for financial development. One is to establish a financial ecological environment evaluation and assessment mechanism. The main indicators are the default rate of loan customers, the rate of nonperforming financial assets, the completion rate of financial disputes, and the level of credit intermediary services to establish evaluation standards. The improvement of the financial ecological environment will be included in the assessment scope of government performance. The second is to combat untrustworthy behaviors in the eyes of enterprises and legal representatives who maliciously evade bank debts and strengthen the role of news media. The government must further strengthen financial opening up based on the principles of building nests and attracting phoenixes, mutual benefit, and common development, so as to encourage foreign capital to expand business and form a trend of interaction between financial and economic development.

This work uses an approximate algorithm based on dynamic relaxation factors to solve this model. The particle structure adopts tree coding. In order to avoid the phenomenon that the particle algorithm accumulates prematurely or falls to the local optimum, a dynamic relaxation approach algorithm based on the current physical condition is proposed. The fitness function is combined with the dynamic relaxation factor to perform corrective functions to optimize particle search. Optimize speed, enhance the local search ability of each particle, and improve the convergence of the particle. After assessing the impact of a sustainable supply chain on economic, environmental, and social factors, the experiment looked at the best cooperation options for a sustainable supply chain, effectively reducing environmental pollution and avoiding overcapacity in the supply chain.

\section{Data Availability}

The data that support the findings of this study are available from the corresponding author upon reasonable request.

\section{Conflicts of Interest}

The authors declare that they have no conflicts of interest.

\section{References}

[1] C. Bals, "Toward a supply chain finance (SCF) ecosystem proposing a framework and agenda for future research," Journal of Purchasing and Supply Management, vol. 25, no. 2, pp. 105-117, 2019.

[2] R. Azzi, R. K. Chamoun, and M. Sokhn, "The power of a blockchain-based supply chain," Computers \& Industrial Engineering, vol. 135, pp. 582-592, 2019.

[3] C. S. Lin and C. Y. Lin, "Constructing a network evaluation framework for improving the financial ecosystem in smallmedium size firms," Technological and Economic Development of Economy, vol. 24, no. 3, pp. 893-913, 2018.

[4] V. Puri, S. Jha, R. Kumar et al., "A hybrid artificial intelligence and internet of things model for generation of renewable resource of energy," IEEE Access, vol. 7, no. 1, pp. 111181111191, 2019.

[5] M. Papert and A. Pflaum, "Development of an ecosystem model for the realization of Internet of Things (IoT) services in supply chain management," Electronic Markets, vol. 27, no. 2, pp. 175-189, 2017.

[6] Y. Chen, W. Zheng, W. Li, and Y. Huang, "The robustness and sustainability of port logistics systems for emergency supplies from overseas," Journal of Advanced Transportation, vol. 2020, Article ID 8868533, 10 pages, 2020.

[7] H. J. Stolze, D. A. Mollenkopf, and D. J. Flint, "What is the right supply chain for your shopper? Exploring the shopper service ecosystem," Journal of Business Logistics, vol. 37, no. 2, pp. 185-197, 2016.

[8] V. Christensen, J. Steenbeek, and P. Failler, "A combined ecosystem and value chain modeling approach for evaluating societal cost and benefit of fishing," Ecological Modelling, vol. 222, no. 3, pp. 857-864, 2011.

[9] V. De Souza, J. Bloemhof-Ruwaard, and M. Borsato, "Exploring ecosystem network analysis to balance resilience and performance in sustainable supply chain design," International Journal of Advanced Operations Management, vol. 11, no. 1/2, pp. 26-45, 2019.

[10] D. Weimer, B. Scholz-Reiter, and M. Shpitalni, "Design of deep convolutional neural network architectures for automated feature extraction in industrial inspection," Cirp Annals Manufacturing Technology, vol. 65, no. 1, pp. 417-420, 2016.

[11] W. Wu, S. An, C. H. Wu, S. B. Tsai, and K. Yang, "An empirical study on green environmental system certification affects financing cost of high energy consumption enterprises-taking metallurgical enterprises as an example," Journal of Cleaner Production, vol. 244, p. 118848, 2020. 
[12] B. C. Boehmke and B. T. Hazen, "The future of supply chain information systems: the open source ecosystem," Global Journal of Flexible Systems Management, vol. 18, no. 2, pp. 163168, 2017.

[13] W. Scacchi and T. A. Alspaugh, "Securing software ecosystem architectures: challenges and opportunities," IEEE Software, vol. 36, no. 3, pp. 33-38, 2019.

[14] P. Hu, F. Wu, J. Peng, Y. Bao, F. Chen, and D. Kong, "Automatic abdominal multi-organ segmentation using deep convolutional neural network and time-implicit level sets," International Journal of Computer Assisted Radiology and Surgery, vol. 12, no. 3, pp. 399-411, 2017.

[15] S. B. Larsen, D. Masi, D. C. Feibert, and P. Jacobsen, "How the reverse supply chain impacts the firm's financial performance: a manufacturer's perspective," International Journal of Physical Distribution and Logistics Management, vol. 48, no. 3, pp. 284-307, 2018.

[16] E. Kopanaki, P. Karvela, and N. Georgopoulos, "From traditional interorganisational systems to cloud-based solutions: the impact on supply chain flexibility," Journal of Organizational Computing and Electronic Commerce, vol. 28, no. 4, pp. 334-353, 2018.

[17] M. A. El Aziz, A. M. Hemdan, A. A. Ewees et al., "Prediction of biochar yield using adaptive neuro-fuzzy inference system with particle swarm optimization," in 2017 IEEE PES PowerAfrica, pp. 115-120, Accra, Ghana, June 2017.

[18] I. D. Blackman, C. P. Holland, and T. Westcott, "Motorola's global financial supply chain strategy," IEEE Engineering Management Review, vol. 45, no. 1, pp. 137-137, 2017.

[19] M. Abdel-Basset, G. Manogaran, and M. Mohamed, "Internet of Things (IoT) and its impact on supply chain: a framework for building smart, secure and efficient systems," Future Generation Computer Systems, vol. 86, pp. 614-628, 2018.

[20] G. K. Mweshi and K. Sakyi, "Building sustainable supply chain investment decisions through financial analysis -case study of Lusaka SMEs," American Scientific Research Journal for Engineering, Technology, and Sciences, vol. 59, no. 1, pp. 81-92, 2019.

[21] K. Kok, S. Pedde, M. Gramberger, P. A. Harrison, and I. P. Holman, "New European socio-economic scenarios for climate change research: operationalising concepts to extend the shared socio-economic pathways," Regional Environmental Change, vol. 19, no. 3, pp. 643-654, 2019.

[22] N. Harring, S. C. Jagers, and S. Matti, "The significance of political culture, economic context and instrument type for climate policy support: a cross-national study," Climate Policy, vol. 19, no. 5, pp. 636-650, 2019.

[23] S.-B. Tsai, R. Saito, Y.-C. Lin, Q. Chen, and J. Zhou, "Discussing measurement criteria and competitive strategies of green suppliers from a green law perspective," Proceedings of the Institution of Mechanical Engineers, Part B: Journal of Engineering Manufacture, vol. 229, Supplement 1, pp. 135-145, 2015.

[24] G. I. Bischi, U. Merlone, and E. Pruscini, "Evolutionary dynamics in club goods binary games," Journal of Economic Dynamics and Control, vol. 91, pp. 104-119, 2018.

[25] A. C. C. Lu, D. Gursoy, and G. del Chiappa, "The influence of materialism on ecotourism attitudes and behaviors," Journal of Travel Research, vol. 55, no. 2, pp. 176-189, 2016.

[26] J.-Y. Yeh and C.-H. Chen, "A machine learning approach to predict the success of crowdfunding fintech project," Journal of Enterprise Information Management, 2020.
[27] C. Liston-Heyes and D. A. Vazquez Brust, "Environmental protection in environmentally reactive firms: lessons from corporate Argentina," Journal of Business Ethics, vol. 135, no. 2, pp. 361-379, 2016.

[28] A. McMullan and A. Majumdar, "Assessing the impact of travel path choice on London's rail network using an automatic fare collection system," Transportation Research Record, vol. 2274, no. 1, pp. 154-163, 2018.

[29] N. Tajbakhsh, J. Y. Shin, S. R. Gurudu et al., "Convolutional neural networks for medical image analysis: full training or fine tuning?," IEEE Transactions on Medical Imaging, vol. 35, no. 5, pp. 1299-1312, 2016.

[30] M. Leroy, "Delivering on environmental commitments? Guidelines and evaluation framework for an "On-board" approach,” Sécheresse, vol. 23, no. 3, pp. 185-195, 2016.

[31] A. Veipa, V. Kirsanovs, and A. Barisa, "Techno-economic analysis of biofuel production plants producing biofuels using Fisher Tropsch synthesis," Environmental and Climate Technologies, vol. 24, no. 2, pp. 373-387, 2020.

[32] Y. Bai and X. Yang, "The research on the economic effect of market-based environmental policy instruments," Open Journal of Social Sciences, vol. 4, no. 4, pp. 38-47, 2016.

[33] I. Bouwma, D. Liefferink, R. Van Apeldoorn, and B. Arts, "Following old paths or shaping new ones in Natura 2000 Implementation? Mapping path dependency in instrument choice," Journal of Environmental Policy \& Planning, vol. 18, no. 2, pp. 214-233, 2016.

[34] P. R. Croes and W. J. V. Vermeulen, "In search of income reference points for SLCA using a country level sustainability benchmark (part 2): fair minimum wage. A contribution to the Oiconomy project," International Journal of Life Cycle Assessment, vol. 21, no. 3, pp. 363-377, 2016.

[35] M. K. M. Singh, "Socio-economic, environmental and personal factors in the choice of country and higher education institution for studying abroad among international students in Malaysia," International Journal of Educational Management, vol. 30, no. 4, pp. 505-519, 2016. 